\title{
Article@Virology
}

\section{Analysis Strategies for Rabies Prevention and Control in China}

Jia Li, Leitai Shi, Yunpeng Wang, Shouchun Cao*

National Institute for Food and Drug Control, Beijing, 102629, P. R. China

\begin{abstract}
Objective To analyze the current epidemiological characteristics of rabies in China, grasp the law of epidemic changes, and provide scientific basis for rabies prevention and control strategies in China. Methods The data of human rabies prevention and control in China were systematically collected, combined with the genetic sequences of rabies virus street virus epidemic strains in various provinces of China, and statistical and molecular biological methods were used for statistics and analysis. Results The number of rabies cases and deaths in China has decreased year by year. At present, the main method of human rabies transmission in China is dog injury. Post-exposure treatment showed an increase in the rate of self-treatment, a decline in the overall immunity rate of the vaccine, and an increase in the rate of passive immunity. Human rabies vaccines are currently available to meet vaccination needs. In addition, the immune rates of dogs and cats did not change significantly. The rabies street virus strains in China are mainly divided into 6 species including China I-China VI. Conclusion In terms of infrastructure, it is still necessary to increase the number of primary clinics, improve the professional capabilities of medical staff, and strengthen publicity and education on rabies prevention and treatment. In the areas with high incidence of cases, vaccines similar to street strain should be selected for immunization of human and animal.
\end{abstract}

Copyright@2012-2020 Published by Hong Kong Institute of Biologicals Standardization Limited. All rights reserved.

\footnotetext{
Article history:Submitted: 30/10/2019; Revised: 25/11/2019; Accepted: 29/11/2019

DOI:10.21092/jav.v8i4.115
}

Key Words: Rabies, Prevention and Control

\footnotetext{
* Corresponding author, ph.D, Major in Immunology

E-mail: caosc@nifdc.org.cn
} 


\section{Introduction}

Rabies is an acute fatal zoonotic disease caused by rabies virus infection. Rabies virus can be transmitted to humans through mucous membranes or damaged skin through the saliva of infected animals, causing human rabies $^{[1]}$. Rabies is widely distributed throughout the world, and human rabies occurs on all continents except Antarctica. Currently, approximately 59,000 people die from rabies in more than 150 countries worldwide each year, with approximately $95 \%$ of cases occurring in Africa and $\mathrm{Asia}^{[2-4]}$. The clinical features of rabies are mainly manic and paralytic ${ }^{[5]}$, and the number of incubation periods in China is 66 days $^{[6]}$.

In China, rabies has been classified as a Class B notifiable infectious disease in 1955 and has experienced three epidemic peaks so far, occurring in the mid 1950s (the highest peak in 1957 was 1942 cases), and in the early 1980s (1981 7037 cases reached the highest peak in the year), early 21 st century (3300 cases reached the highest peak in $2007)^{[7,8]}$. As of now, rabies cases have been reported in 31 provinces in China. Southern, eastern and central provinces are the regions with high incidence of rabies, especially in Guangdong, Guangxi, Guizhou, and Hunan $^{[9,10]}$, and there are more cases of men than women, 15 years old. The following children and the elderly over the age of 50 are the high-risk groups ${ }^{[11]}$.

As a vaccine preventable disease, rabies can be divided into pre-exposure prevention and post-exposure prevention (PEP, Post exposure prophylaxis). The general population should take appropriate preventive measures according to the level of exposure after exposure to rabies virus: Level I exposure does not require treatment, level II exposure requires immediate wound management (rinsing, disinfection) and rabies vaccine, and level III exposure requires immediate wounding In addition to handling and vaccination against rabies, passive immunizations are also required ${ }^{[12]}$.

This paper intends to systematically sort out the current data on rabies prevention and control in China, explore the internal relationship between the data, and provide data support for the next step in China's rabies prevention and control strategy.

\section{Materials and Method}

1.Data source

1.1 Annual rabies data in China: Public Health Scientific the Data-Center of China Public Health Science from National Population and Health Science Data Sharing Platform.

1.2 Annual post-exposure treatment information for rabies in China: epidemic data from Chinese Infectious Disease Surveillance Reporting System, sentinel surveillance system in 6 provinces 
(Hunan, Guangxi, Anhui, Guizhou, Jiangsu and Shandong) and 15 national surveillance Point (Anlong County, Suiyang County and Dushan County, Guizhou Province, Guilin City, Yulin City, Qinzhou City, and Guigang City, Guangxi Zhuang Auto-nomous Region, Shaoyang City, Yongzhou City, and Hengyang City, Hunan Province, Fuyang City, Mengcheng County, Anhui Province And Lujiang County, Yancheng City, Jiangsu Province, and Linyi City, Shandong Province).

\subsection{China Annual Rabies Vaccine Issuance Data: National Institute for Food and Drug Control(NIFDC)}

1.4 The glycoprotein nucleic acid sequence of street virus isolates in China in recent years: GenBank

\section{Result}

1. Overview of rabies epidemic

\subsection{Death cases of rabies from 1996 to 2019}

Statistics on the overall rabies epidemic situation in China from 1996 to October 2019. The total number of reported cases of rabies in the country reached 31,943 . Among them, the overall rabies epidemic in China from 1996 to 2007 showed an upward trend. Since 2008, the number of reported rabies cases in China has continued to decline. In recent years, the annual number of deaths has been less than 500, which has fallen by over $80 \%$ compared with the high value in 2007 .

\subsection{Monthly Rabies Cases}

Statistics on rabies deaths in each month from 2015 to 2019. Rabies deaths are reported all year round, especially in summer (June-August) and winter (November-January). In recent years, except for individual months (such as the summer of 2016), the number of rabies deaths has been decreasing month-on-month. With reference to the median incubation period of rabies in China, which is 66 days, the high incidence of rabies injury in China is April-June and September-November.

1.3 Population distribution of rabies deaths

In recent years, the majority of rabies deaths are farmers, followed by students and scattered children. There are more male cases than female cases, and the age distribution has a bimodal state, that is, 0-14 and 55 years old, And more than middle-aged and older people. The specific statistical results are shown in the table below.

\subsection{Regional distribution of rabies deaths}

As of 2018, rabies deaths have been reported in China's 31 provinces. The number of cases in the central and southern regions in China is relatively high, and the situation is highly distributed across the country. With the exception of Hunan and Hubei provinces, 


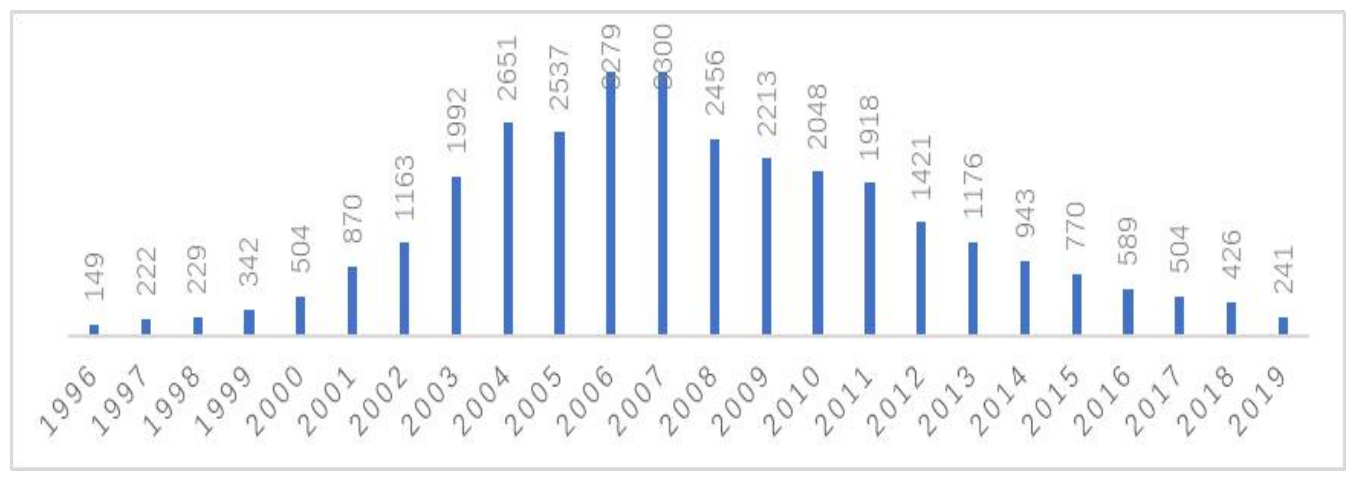

Figure 1: Reported cases of rabies in China from 1996 to 2019 (Oct)

Note: The statistical time is as of October 2019



Figure 2: Reported monthly cases of rabies in China from 2015 to 2019 (Oct)

Note: The statistical time is as of October 2019

Tab1: Population distribution of rabies deaths in recent years

\begin{tabular}{ccccc}
\hline Year & $\begin{array}{c}\text { Ratio of } \\
\text { Male(\%) }\end{array}$ & $\begin{array}{c}\text { Ratio of } \\
\text { Farmer }(\%)\end{array}$ & $\begin{array}{c}\text { Ratio of } \\
\text { Students }(\%)\end{array}$ & $\begin{array}{c}\text { Ratio of Scattered } \\
\text { Children }(\%)\end{array}$ \\
\hline 2012 & 72 & 70.9 & 8.3 & 5.8 \\
2013 & 69 & 70.82 & 8.87 & 6.31 \\
2015 & 73 & 75 & 6.37 & 5.87 \\
2016 & 68 & 68.94 & 9.01 & 5.75 \\
2017 & 71 & 76.74 & 6.4 & 4.46 \\
\hline
\end{tabular}

Copyright@2012-2020 Published by Hongkong Institute of Biologicals Standardization Limited. All rights reserved. 
where there has been no significant decrease in deaths, the incidence of deaths in all other provinces has been decreasing year by year, despite occasional recurrences. The cases of all provinces were sporadic and there were no concentrated outbreaks. In 2018, the top five provinces reporting deaths were Hunan Province (78 cases / 18.5\%), Henan Province (42 cases / 10.0\%), Guangxi Province and Hubei Province (34 cases / 8.1\%), Guizhou Province (31 cases). /7.4\%). From 2013 to 2018, the provinces with the largest decline in reported deaths were Hainan (100\%), Guangdong (87.14\%), Shandong (85.45\%), Hebei $(83.82 \%)$, and Shanxi $(83.33 \%)$. The median decline rate in each province was $68.7 \%$. The specific data is shown in Table 2.

\section{Post-exposure treatment of rabies}

Systematic publication of publicly available data ${ }^{[13-17]}$ of epidemic data from Chinese Infectious Disease Surveillance Reporting System, From 2012 to 2017 (there is no 2014 data), the number of annual attendances at 15 national monitoring sites in 6 provinces is shown in Figure 3, and the attending population showed a rising trend year by year. The system collects data on the number of cases at each monitoring point in the provinces and age groups of mainland China, including rabies cases, post-exposure prophylaxis and host animal surveys. From the data, we can see that there are more men than women in animal-injured patients in
China. Domestic dogs cause injuries, and cat-injured patients are increasing year by year. Patient exposure levels are mostly grades II and III. During the wound treatment stage, the proportion of outpatient treatment has gradually increased, although patients who have completed the full immunization in the past two years The proportion has declined, but the proportion of patients who have completed passive immunization, that is, injection immunization, continues to increase. In terms of detection of host animals, the average density of dogs and cats causing major injuries remained stable, but the average immunization rate of dogs decreased. See Table 3 for specific summary data.

3. Issuance of human rabies vaccine batch Systematically sort out the number of rabies vaccines issued by human vaccine manufacturers (counts) announced by NIFDC from 2012 to November 2019, and calculate the annual and monthly human rabies vaccines according to the packaging specifications of each manufacturer Number of people issued. From the data, the total number of rabies vaccines for human use fluctuates from year to year, ranging from 11 million to 17 million. The seasonal rabies vaccine supply also fluctuates due to seasonal and corporate production schedules. The annual and monthly issuances are shown in Figure 4 and Figure 5, respectively. 
Table 2: Reported Death cases of rabies in Province from 2013 to 2018

\begin{tabular}{|c|c|c|c|c|c|c|c|}
\hline \multirow{2}{*}{ Province } & \multicolumn{6}{|c|}{ Death Case } & \multirow{2}{*}{$\begin{array}{c}\text { Decline } \\
\text { Rate } \\
(\%)\end{array}$} \\
\hline & 2013 & 2014 & 2015 & 2016 & 2017 & 2018 & \\
\hline Guangxi & 161 & 121 & 78 & 82 & 41 & 34 & 78.88 \\
\hline Guangdong & 140 & 85 & 48 & 44 & 23 & 18 & 87.14 \\
\hline Guizhou & 84 & 70 & 62 & 51 & 26 & 31 & 63.10 \\
\hline Hunan & 83 & 65 & 75 & 64 & 71 & 78 & 6.02 \\
\hline Henan & 81 & 78 & 78 & 82 & 52 & 42 & 48.15 \\
\hline Yunnan & 79 & 73 & 57 & 48 & 32 & 21 & 73.42 \\
\hline Hebei & 68 & 56 & 44 & 31 & 8 & 11 & 83.82 \\
\hline Sichuan & 67 & 45 & 36 & 20 & 27 & 21 & 68.66 \\
\hline Shanxi & 66 & 43 & 25 & 26 & 17 & 11 & 83.33 \\
\hline Shandong & 55 & 32 & 37 & 25 & 23 & 8 & 85.45 \\
\hline Shananxi & 49 & 31 & 25 & 14 & 17 & 18 & 63.27 \\
\hline Hubei & 38 & 41 & 37 & 32 & 39 & 34 & 10.53 \\
\hline Jiangsu & 37 & 39 & 34 & 44 & 21 & 22 & 40.54 \\
\hline Anhui & 31 & 31 & 34 & 23 & 39 & 17 & 45.16 \\
\hline Chongqing & 29 & 30 & 27 & 21 & 24 & 18 & 37.93 \\
\hline Hainan & 29 & 21 & 8 & 4 & 5 & 0 & 100.00 \\
\hline Jiangxi & 17 & 13 & 10 & 11 & 15 & 13 & 1 \\
\hline Inner Mongolia & 13 & 6 & 7 & 7 & 2 & 2 & / \\
\hline Gansu & 9 & 4 & 11 & 8 & 9 & 1 & / \\
\hline Zhejiang & 8 & 9 & 8 & 19 & 14 & 13 & l \\
\hline Ningxia & 8 & 14 & 8 & 2 & 1 & 1 & l \\
\hline Beijing & 7 & 7 & 10 & 3 & 3 & 2 & l \\
\hline Tianjin & 4 & 5 & 2 & 5 & 2 & 0 & / \\
\hline Liaoning & 4 & 2 & 3 & 0 & 0 & 0 & l \\
\hline Fujian & 2 & 0 & 3 & 1 & 2 & 0 & l \\
\hline Shanghai & 1 & 3 & 0 & 1 & 2 & 5 & l \\
\hline Qinghai & 1 & 0 & 0 & 1 & 1 & 0 & l \\
\hline Heilongjiang & 1 & 0 & 0 & 0 & 0 & 0 & / \\
\hline Jilin & 0 & 0 & 1 & 0 & 0 & 0 & l \\
\hline Xinjiang & 0 & 0 & 0 & 1 & 0 & 0 & I \\
\hline Tibet & 0 & 0 & 1 & 1 & 1 & 0 & 1 \\
\hline
\end{tabular}

Note: The decline rate only counts provinces with more than 20 deaths in 2013.

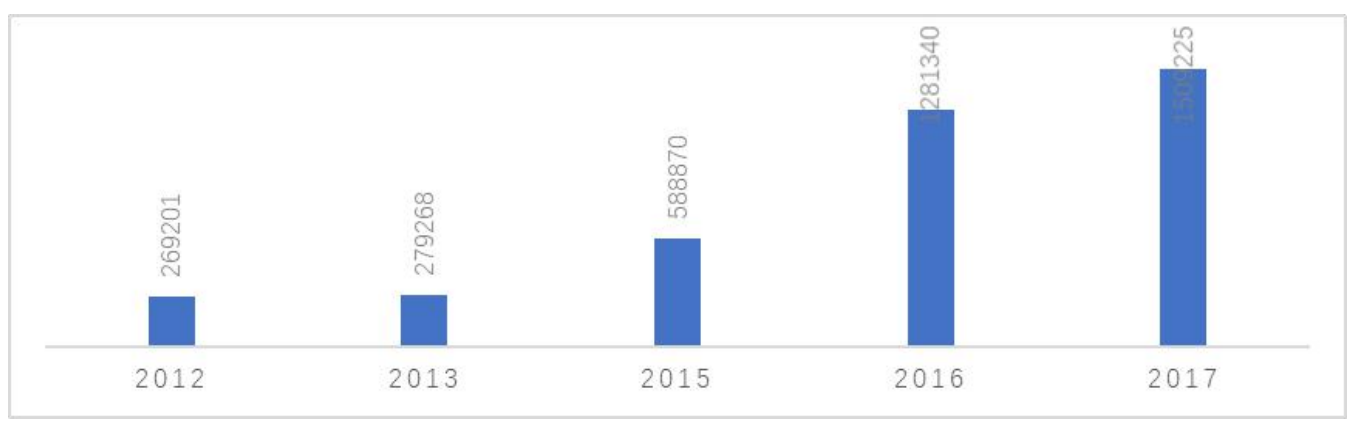

Figure 3: Annual number of patients visited at national monitoring sites 
Table 3: Data summary of 6 provincial monitoring points

\begin{tabular}{|c|c|c|c|c|c|c|}
\hline \multirow{2}{*}{ Category } & \multirow{2}{*}{ Index } & \multicolumn{5}{|c|}{ Year } \\
\hline & & 2012 & 2013 & 2015 & 2016 & 2017 \\
\hline Sex & Male to female ratio & 1.3 & 1.3 & 1.2 & 1.2 & 1.2 \\
\hline \multirow{2}{*}{ Injury animal } & $\operatorname{Dog}(\%)$ & 82 & 80.37 & 77.81 & 79.48 & 76.32 \\
\hline & Cat $(\%)$ & 12 & 12.17 & 14.94 & 13.03 & 14.72 \\
\hline \multirow{2}{*}{$\begin{array}{l}\text { Injurious } \\
\text { animal } \\
\text { source }\end{array}$} & Domestic $(\%)$ & 62.5 & 70.07 & 64.14 & 63.00 & 71.92 \\
\hline & Stray (\%) & 32.1 & 28.52 & 30.84 & 29.50 & 22.81 \\
\hline \multirow{3}{*}{$\begin{array}{l}\text { Exposure } \\
\text { grading }\end{array}$} & $\mathrm{I}(\%)$ & 7 & 7.07 & 7.41 & 6.67 & 5.71 \\
\hline & II $(\%)$ & 50 & 51.51 & 50.83 & 48.57 & 48.25 \\
\hline & III $(\%)$ & 43 & 41.42 & 41.74 & 44.76 & 46.04 \\
\hline \multirow{3}{*}{$\begin{array}{l}\text { Wound } \\
\text { treatment }\end{array}$} & Self $(\%)$ & 21 & 18.18 & / & 26.56 & 32.18 \\
\hline & Outpatient $(\%)$ & 79 & 81.79 & / & 73.04 & 67.25 \\
\hline & none $(\%)$ & 1 & 0.03 & 1 & 0.4 & 0.57 \\
\hline & Full immunization (\%) & 89 & 87.81 & 90.51 & 77.66 & 76.69 \\
\hline \multirow[t]{2}{*}{ Vaccination } & Not Full（\%) & 10 & l & / & 22.25 & 23.16 \\
\hline & none $(\%)$ & 1 & 1 & 1 & 0.09 & 0.05 \\
\hline $\begin{array}{c}\text { Passive } \\
\text { immunization }\end{array}$ & $\begin{array}{c}\text { Rabies } \\
\text { immunoglobulin }(\%)\end{array}$ & 16 & 13.45 & 12.95 & 28.89 & 31.79 \\
\hline \multirow{4}{*}{$\begin{array}{l}\text { Host animal } \\
\text { monitoring }\end{array}$} & $\begin{array}{l}\text { Average dog density } \\
\text { (amount/100Persons) }\end{array}$ & 6.6 & 6.7 & 6.94 & 7.03 & 7.2 \\
\hline & $\begin{array}{c}\text { Dog average } \\
\text { immunization rate }(\%)\end{array}$ & 41.4 & 43.63 & 43.34 & 37.64 & 30.89 \\
\hline & $\begin{array}{l}\text { Average cat density } \\
\text { (amount/100Persons) }\end{array}$ & 1.3 & 1.49 & 2.09 & 1.48 & 1.63 \\
\hline & $\begin{array}{c}\text { Cat average } \\
\text { immunization rate }(\%)\end{array}$ & 14.5 & 11.91 & 0.24 & 11.3 & 11.02 \\
\hline
\end{tabular}

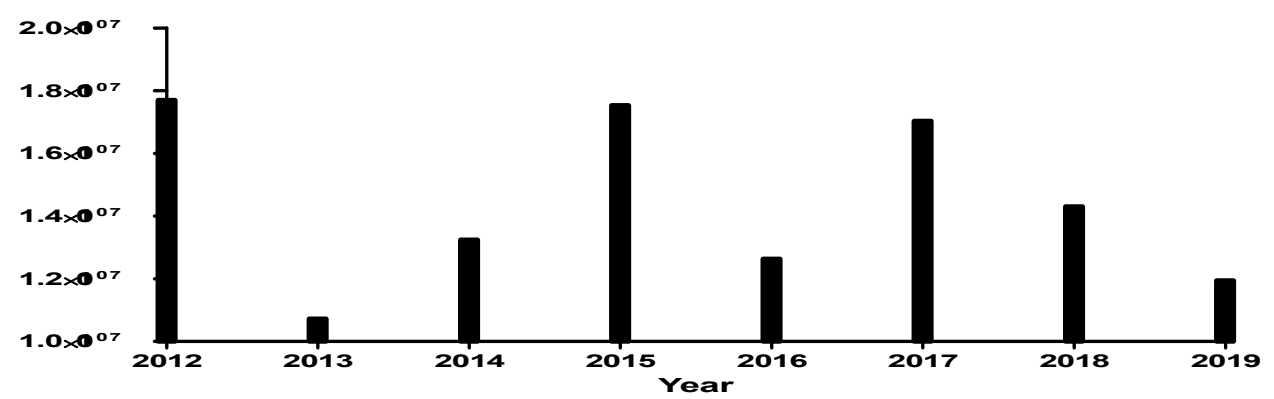

Figure 4: Summary of human rabies vaccine issuances (person servings) for each year from 2012 to November 2019

Copyright@2012-2020 Published by Hongkong Institute of Biologicals Standardization Limited. All rights reserved. 


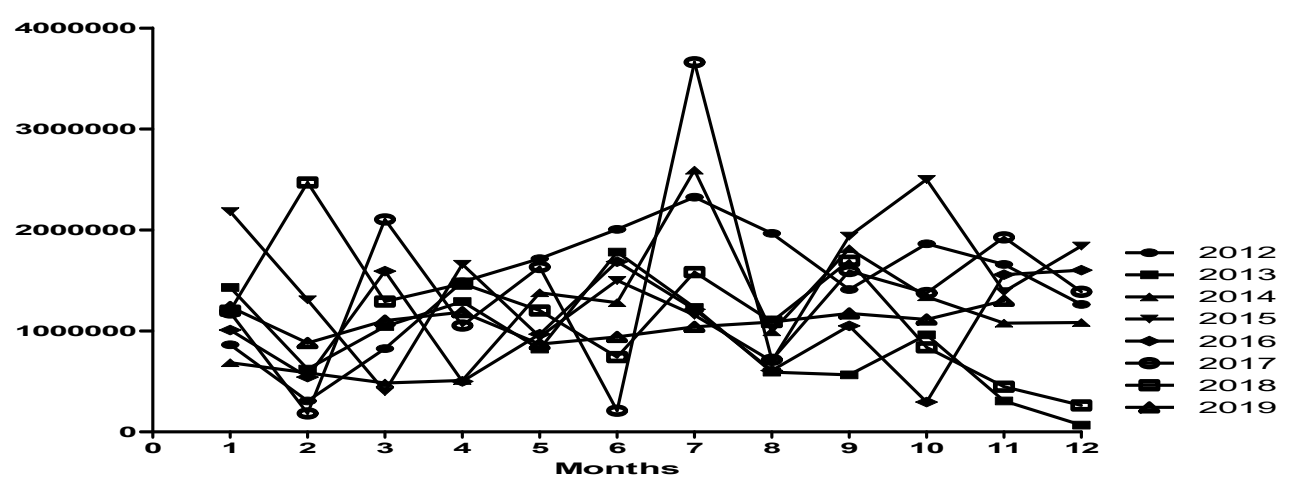

Figure 5: Summary of monthly rabies vaccine issuances (person servings) from 2012 to November 2019

4. Molecular epidemic trend of rabies virus street virus isolated in China in recent years

Systematic collection of sequences of glycoprotein ORF coding regions of serum rabies virus street virus isolated from various provinces and cities in recent years. The hosts involved mainly include patients, domestic pets (dogs, cats), stray dogs, domestic animals (pigs, cattle, sheep, etc.), Wild animals (rats, foxes, etc.), and systematically collected the glycoprotein ORF coding region sequences of human rabies vaccines and veterinary rabies vaccine production strains in China, plotted evolutionary trees together, and analyzed rabies street virus epidemic trends in various regions. According to the evolutionary tree analysis, although the street virus strains and vaccine-producing strains collected in China are serum type I genotype I viruses, viruses isolated from different regions and different hosts can be divided into 6 populations $^{[18]}$, which are China I-China VI, in which the various groups can still be divided into several subgroups. Among them, ChinaI, II, V, and VI populations are relatively close to each other. The virus isolated from dogs and patients in most areas of China belongs to this population, and is closely related to the vaccine production strain CTN-1V. More recently, most of the viruses isolated from wild animal hosts in northwestern China belong to this population, and they are closely related to vaccine strains $\mathrm{aG}$, PM1503, PV2061, FluryLEP, ERA, and SAD B19. In addition, similar street populations of street viruses For example, China I, II, V, and VI street virus epidemic strains exist in Guangxi Zhuang Autonomous Region, and China III and IV street virus epidemic strains exist in 
Inner Mongolia Autonomous Region. The specific analysis results are shown in Figure 6. The representative strain sequences in various groups were selected and analyzed for homology with the current strains for the production of human and canine rabies vaccines in China. The specific results are shown in Table 4.

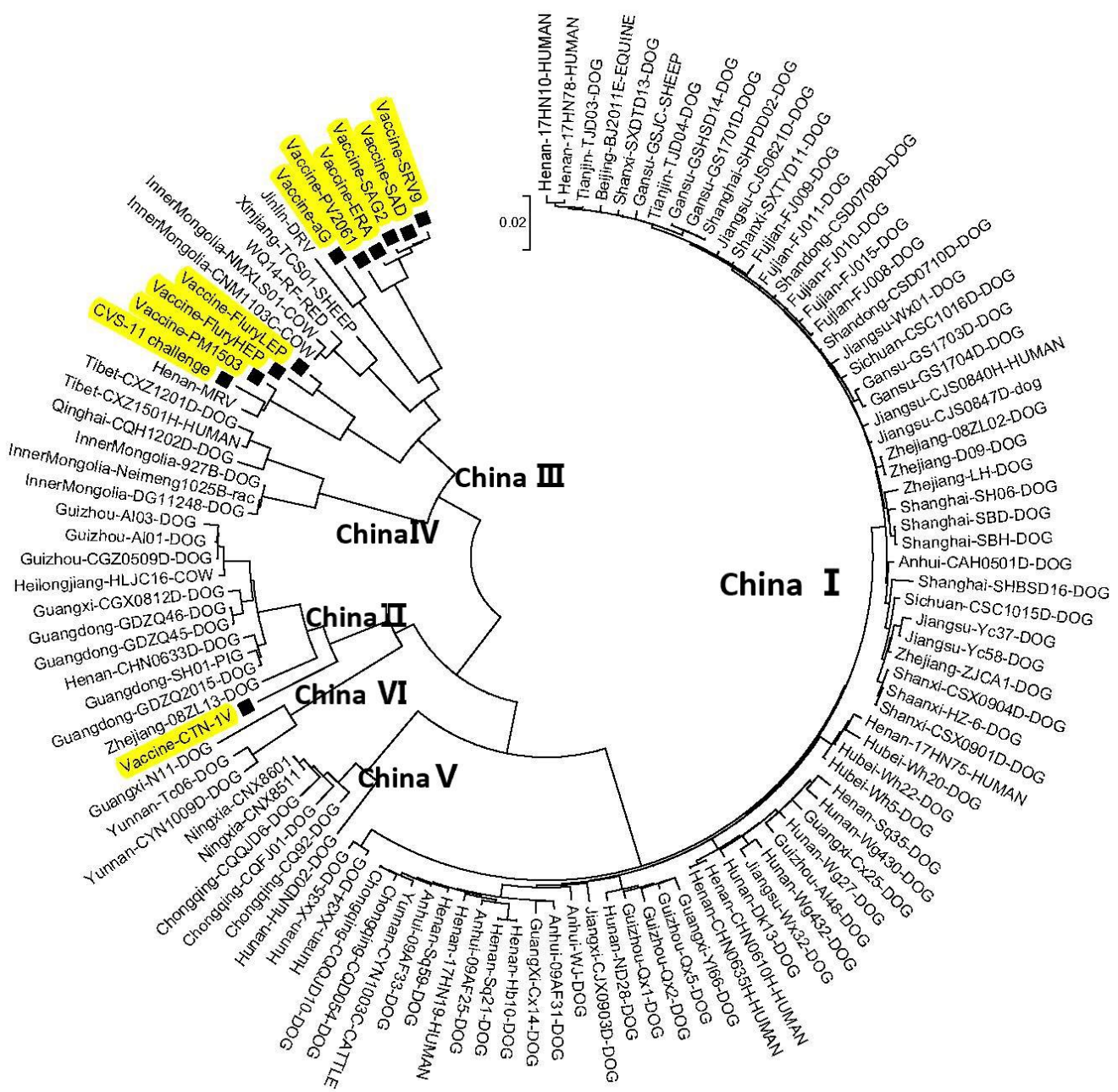

Figure 6: Rabies virus glycoprotein evolution tree 
Table 4: Summary of glycoprotein similarities between vaccine strains and Chinese street virus epidemic strains

\begin{tabular}{|c|c|c|c|c|c|c|c|c|}
\hline \multirow{2}{*}{\multicolumn{2}{|c|}{ Strain }} & \multicolumn{7}{|c|}{ Similarity(\%) } \\
\hline & & CVS-11 & ChinaI & ChinaII & ChinaIII & ChinaIV & ChinaV & ChinaVI \\
\hline \multirow{5}{*}{$\begin{array}{c}\text { For } \\
\text { Human } \\
\text { Use }\end{array}$} & CTN-1V & 83 & 88 & 94.8 & 85.1 & 84.4 & 89 & 89.1 \\
\hline & $\mathrm{aG}$ & 88.2 & 82.3 & 83.9 & 91.5 & 85.8 & 82.9 & 82.6 \\
\hline & PM1503 & 98.4 & 83.2 & 84 & 90.3 & 87.7 & 84.4 & 93.6 \\
\hline & PV2061 & 88.4 & 83.3 & 83.9 & 90.2 & 87.8 & 83.5 & 82.9 \\
\hline & FluryLEP & 93.5 & 83.9 & 85.3 & 90.9 & 88.2 & 85.7 & 84.4 \\
\hline \multirow{2}{*}{$\begin{array}{c}\text { For } \\
\text { Canine } \\
\text { Use }\end{array}$} & ERA & 88.4 & 83.1 & 83.7 & 92.4 & 87.2 & 84.1 & 82.7 \\
\hline & SAD B19 & 88.2 & 82.9 & 83.7 & 91.2 & 87.7 & 83.3 & 83.6 \\
\hline
\end{tabular}

\section{Discussion}

In recent years, the number of rabies cases in China has been decreasing year by year. Although there were more than 3,000 cases of rabies deaths in China in 2007, as of October 2019, the annual death rate has been less than 300 cases, and rabies prevention and control measures have achieved great results. A systematic analysis of monthly statistics of rabies deaths in each year shows that summer (June-August) and winter (November-January) are the high incidence of rabies deaths each year. Given that the median incubation period for rabies in China is 66 Therefore, the high incidence of rabies injury in China is spring (April-June) and autumn (September-November), which overlaps with the estrous period (spring, autumn) of conventional dogs. It is speculated that Dogs in estrus are more aggressive and are more likely to attack people in close contact.
From the statistics, we can know that rabies deaths in China have been dominated by farmers, students, and scattered children for many years, and most of them are aged $0-14$ and over 55 years. Therefore, the state still needs to increase financial investment, strengthen popularization and publicity Knowledge of rabies prevention and control, increase the number of grass-roots clinics and continue to improve the capacity and professional ability of grass-roots clinics, to ensure that people who have been bitten receive timely, standardized, and complete post-exposure prevention (Postexposure Prophylaxis, PEP), which is important for the prevention of rabies significance.

The general population should take appropriate preventive measures according to the level of exposure after exposure to rabies virus: Level I exposure does not require treatment, level II 
exposure requires immediate wound treatment (rinsing, disinfection) and rabies vaccine, and level III exposure requires immediate wounding In addition to handling and vaccination against rabies, passive immunizations are also required. Compre-hensive analysis of monitoring data from 15 national monitoring points in 6 provinces (Guizhou, Guangxi, Hunan, Anhui, Jiangsu, Shandong) in China in recent years. The exposure levels of injured patients are mostly level II and level III. The propor-tion of wound treatment is increasing year by year. In China, there are more male-injured patients than females, and most of them are domestic dogs. Cat-injured patients are also increasing year by year. The patient exposure levels are mostly II and III. During the wound treatment stage, Hunan Province and Patients in Guangxi Zhuang Autonomous Region have a higher proportion of self-treatment. In the vaccine immunization stage, the overall immuniza-tion rate in Guangxi Zhuang Autonomous Region and Shandong Province is low, less than $80 \%$. In terms of passive immunization, the passive immunization rates in Guangxi Zhuang Autonomous Region and Hunan Province Higher, all more than 50\%. According to the annual number of rabies deaths in each province, it is known that the number of deaths in Hunan Province has maintained a high level so far. Taking the data from 2017 as an example, among the annual death rate, Hunan Province ranks first in all provinces in the country with 71 cases, accounting for $13.73 \%$ of the total national deaths.
Surveillance data reported by monitoring points in various provinces shows that, despite the overall immunity rate in Hunan Province, (99.43\%) ranks first in 6 provinces, but the proportion of patients who treat wounds in outpatient clinics is only $6.33 \%$, which is significantly lower than the data in the remaining 5 provinces (all of which are not less than $55.41 \%$ ). It can be inferred that after patients suffered exposure injuries, The inability to accept formal wound treatment in the first place may be one of the reasons for the high level of rabies deaths in Hunan Province.

Vaccination is an important part of the PEP process. The rabies vaccine for human is inoculated with rabies virus fixed virus into appropriate cells, and is cultured, harvested, concentrated, virus inactivated and purified, and then added with appropriate stabilizers to prevent rabies. At present, the production strains of rabies vaccine for human use in China mainly include CTN-1V strain, aG strain, PV2061 strain and PM1503 strain. The cell matrix mainly includes Vero passage cells, human diploid cells and primary hamster kidney cells. The dosage form involves both lyophilized and injection forms. At present, the rabies vaccine for humans in our country implements a batch management system for the issuance of biological products. The annual issuance volume is between 11 million and 17 million, with a certain volatility. The production of rabies vaccine for human use is hardly affected 
by the season, and it can also be scheduled throughout the year. Although there is a fluctuation in the amount of vaccine issued each month, there is no significant difference. According to the data analysis, although the production of rabies vaccines for human use has declined in 2018 and 2019 , the number of rabies deaths has continued to decline. In addition, the number of vaccines issued in the month of approval and the number of rabies latency periods (the first two cases of rabies deaths) There is no corresponding relationship, and the vaccination rate of the population exposed at the provincial inspection sites in each year reaches more than $99 \%$ (full course + non-full course). Therefore, the current rabies vaccine output can meet the needs of rabies prevention and control in China. Some scholars believe that human rabies vaccine is currently abused in China, but according to data from surveillance sites, a small number of exposed people who are diagnosed with class I exposure die each year from rabies. Due to the high mortality of rabies, clinical needs More skilled medical personnel judge the exposure of the wound and take more stringent measures to prevent and treat rabies.

At present, the results of case investigations at surveillance sites show that the injured animals are mainly dogs, followed by cats, indicating that dogs are still the host animals that cause human rabies in China. $>50 \%$ of the injured animals are domestic animals, followed by stray animals, so it is urgent to strengthen dog immunity and management. Monitoring results show that China still lacks a reasonable and standardized scientific system for dog management. Provinces should attach importance to cooperation with the agricultural and public security departments, strengthen the management of dogs, establish and improve domestic animal file information, and actively promote the use of veterinary vaccines. Although animal epidemic prevention is the way to prevent the transmission of rabies virus at source, and China's urban policies have formulated and planned vaccination programs for domestic dogs, the effectiveness of the measures is not yet available. The data shows that in recent years, the average dog density at each surveillance point has remained stable, but the dog immunity rate has been decreasing year by year, from $43.63 \%$ in 2013 to $30.89 \%$ in 2017 , and the human rabies death rate has also been decreasing year by year. There is no intrinsic correlation between dog immunity and the number of human rabies patients. In addition, the dog immunization rate varies greatly in various regions. Although there are reports in 2013 that the average dog immunization rate at 4 surveillance sites in Guangxi Zhuang Autonomous Region reached $92.12 \%$, the number of rabies 
deaths in Guangxi Zhuang Autonomous Region in 2013 was still 161. Ranked first in each province. Due to the high proportion of domestic animals that are injured in China, in areas with high incidence of rabies, in addition to regular immunization of domestic animals, close contact between humans and animals should be avoided or reduced as much as possible, and wounds should be performed in time after exposure. Handling and vaccination.

At present, the main rabies viruses in China are serotype I and genotype I. Rabies carried by different regions and different hosts can be divided into 6 genera, ChinaI-ChinaVI, among which ChinaI, II, V, and VI populations are closely related, and the virus isolated from dogs and patients in most areas of China belongs to this The populations of ChinaIII and IV populations are relatively close. Most of the viruses isolated from wild animal hosts in northwestern China belong to this population. In addition, there are similar populations of street virus epidemic strains in the same area, such as ChinaI, II, V, The epidemic strains of street virus strains in population VI, and the epidemic strains of China III and IV populations in Inner Mongolia Autonomous Region.

Among vaccines used for vaccine production in China, CTN-1V strain and aG strain were isolated, fixed and adapted by China and can be used to prepare vaccines. Among them, CTN-1V strain was isolated from the brain tissue of Shandong patients, and can be used for preparation after being fixed and passaged. Vero cell vaccine, aG strain virus isolated from Beijing Crazy Dog Brain, can be used to prepare Vero cells and primary hamster kidney cell vaccines after being fixed and passaged; PM1503 and PV2061, both sourced from standard challenge virus (CVS-11) strains In the original French Pasteur strain isolated from Mad Cow, PM1503 strain is currently used for vaccine preparation of human diploid cells and Vero cells, and PV2061 strain is used for vaccine preparation of Vero cells. It is worth mentioning that due to PV2061 The passage history of the strain is different from PM1503 strain. The two viruses are no longer in the same population, while PM1503 strain and CVS-11 strain are still highly homologous and in the same population. PM1503 and PV2061 strains differ not only in their coding regions but also in their non-coding regions. The non-coding regions of the glycoprotein genes of PV2061 have shifted, that is, the non-coding regions after the glycoprotein ORF are removed from the glycoprotein The non-coding region (polyA tail) is placed between the non-coding region of the $\mathrm{G}$ gene and the L gene (see Figure 6). The reason has not yet been verified.

It has been reported in the literature that rabies virus glycoprotein homology 
greater than $74 \%$ has cross-protection. From the analysis of the strain glycoprotein homology, it can be seen that although the street virus strains that are prevalent in various regions of China have species diversity and the closeness of the genetic relationship of virus strains isolated between different hosts is different, the virus sugars of various genera are different. The similarity between the protein and the glycoprotein of the current commercial and canine vaccine production virus species in China are higher than $80 \%$, that is, the vaccine is protective. Among them, the CTN-1V strain is more closely related to China I, II, V, and VI populations than other production strains, and has similar glycoprotein similarity to human rabies virus and canine rabies virus in most regions of China. aG, PM1503, PV2061, FluryLEP, ERA, and SAD B19 are more closely related to China III and IV populations, and are more similar to rabies viruses carried by wild animals in northwest China. In view of the differences in the rabies epidemic in different regions of China, in order to further improve the vaccine immunization effect and reduce the incidence of rabies in regions with high incidence of rabies in our country, each region can adopt the species distribution of the epidemic of the street virus in the region, and select targeted The vaccine is used to immunize people and animals.

In summary, at present, China's rabies prevention and control has achieved phased victory, with annual deaths significantly reduced and showing a downward trend year by year. Although the distribution of rabies in China is still highly distributed, the prevention and control situation in some provinces and cities is still severe. For example, the central and western regions such as Hunan Province, Henan Province, Guangxi Province, Hubei Province and Guizhou Province still have many Reports of rabies cases. Based on the comprehensive situation of the above-mentioned regions, it is necessary to further increase the investment in infrastructure, increase the number of primary clinics, improve the professional and technical capabilities of medical staff, and strengthen publicity and education on rabies prevention.

\section{References}

[1] World Health Organization. Zero by 30: the global strategic plan to end human deaths from dog-mediated rabies by 2030: United Against Rabies Collaboration: first annual progress report: global strategic plan to end human deaths from dog-mediated rabies by 2030[M]//Zero by 30: the global strategic plan to end human deaths from dog-mediated rabies by 2030: United Against Rabies Collaboration: first annual progress report: global strategic plan to end human deaths from dog-mediated rabies by 2030. 2019.

[2] WHO.Rabies: Epidemiology and burden of disease. 2018. Available from: https://www.who.int/rabies/epidemiology/en /. 
[3] Hampson K, Coudeville L, Lembo T, et al. Estimating the global burden of endemic canine rabies[J]. PLoS neglected tropical diseases, 2015, 9(4): e0003709.

[4] World Health Organization. Human rabies: 2016 updates and call for data[J]. Wkly Epidemiol Rec, 2017, 92(7): 77-86.

[5] Hemachudha T, Laothamatas J, Rupprecht C E. Human rabies: a disease of complex neuropathogenetic mechanisms and diagnostic challenges[J]. The Lancet Neurology, 2002, 1(2): 101-109.

[6] Hemachudha T, Ugolini G, Wacharapluesadee $\mathrm{S}$, et al. Human rabies: neuropathogenesis, diagnosis, and management[J]. The Lancet Neurology, 2013, 12(5): 498-513.

[7] Song M , Tang Q, Rayner S, et al. Human rabies surveillance and control in China, 2005-2012[J]. BMC Infectious Diseases, 2014, $14(1): 212$.

[8] Zhou H, Vong S, Liu K, et al. Human Rabies in China, 1960-2014: A Descriptive Epidemiological Study[J]. PLoS Negl Trop Dis. 2016. 10(8): e0004874.

[9] Yao H W, Yang Y, Liu K, et al. The Spatiotemporal Expansion of Human Rabies and Its Probable Explanation in Mainland China, 2004-2013[J]. Plos Negl Trop Dis. 2015. 9(2): e0003502.

[10] Transmission dynamics of re-emerging rabies in domestic dogs of rural China.[J]. PLoS Pathogens, 2018.

[11] Zhou H, Li Y, Chen R F, et al. Technical guideline for human rabies prevention and control (2016)[J]. Zhonghua liu xing bing xue za zhi= Zhonghua liuxingbingxue zazhi, 2016, 37(2): 139-163.
[12] WHO. Rabies vaccines: WHO position paper-- April 2018[J]. Wkly Epidemiol Rec. 2018. 93(16): 201-220.

[13] Zhou H, Li Y, Mu D , et al. [Analysis of epidemiological features of human rabies in China, 2012][J]. Zhonghua liu xing bing xue za zhi $=$ Zhonghua liuxingbingxue zazhi, 2015, 36(3):205-209.

[14] Zhou H, Mou D, Li Y. Epidemiological features and analysis on human rabies in China, 2013[J]. International Journal of Virology, 2015, 3: 145-148.

[15] Liu S Q, Tao X Y, Yu P C. Epidemiological features and analysis on human rabies in 2015[J]. China, 2016, 30(6): 537-540.

[16] Li Y R, Zhu L L, Zhu W Y, et al. Epidemiology of human rabies in China, 2016[J]. Zhonghua liu xing bing xue za zhi= Zhonghua liuxingbingxue zazhi, 2018, 39(1): 40-43.

[17] Liu J J, Duo L, Tao X Y, et al. Epidemiological characteristics of human rabies in China, 2017[J]. Zhonghua liu xing bing xue za zhi $=$ Zhonghua liuxingbingxue zazhi, 2019, 40(5): 526-530.

[18] Yu P C, Tao X Y, Wang L H, et al. Establishment of a Chinese street rabies virus library and its application for detecting neutralizing activity[J]. Infectious diseases of poverty, 2018, 7(1): 117. 